\title{
A unique MUC1-2-VNTR DNA vaccine suppresses tumor growth and prolongs survival in a murine multiple myeloma model
}

\author{
YUHUI WENG ${ }^{1,3}$, LIANG SHAO ${ }^{2}$, HONGMEI OUYANG ${ }^{1,5}$, YUEBO LIU $^{1}$, JIN YAO $^{1}$, HONG YANG $^{1}$, \\ YUNJIAO LUO ${ }^{1,4}$, HAO WANG $^{1}$, ZHENYU ZHAO $^{1}$, HONG MOU $^{1}$, ZEPING ZHOU $^{1}$ and YOU ZHANG ${ }^{1}$ \\ ${ }^{1}$ Department of Hematology, Second Affiliated Hospital of Kunming Medical University, \\ Kunming 650101; ${ }^{2}$ Department of Medicine, The University of Hong Kong, Queen Mary Hospital, \\ Hong Kong; ${ }^{3}$ Department of Hematology, Fuqing Hospital, Fuqing 350300; ${ }^{4}$ Children's Hospital of \\ Kunming, Kunming 650000; ${ }^{5}$ First People's Hospital of Yunnan, Kunming 650000, P.R. China
}

Received November 17, 2011; Accepted February 3, 2012

DOI: 10.3892/or.2012.1707

\begin{abstract}
Multiple myeloma (MM) is a clonal B-cell malignancy charactered by the aberrant proliferation of malignant plasma cells in the bone marrow. MM is still an incurable malignancy. In this regard, novel treatments are urgently required. MUC1 (mucin 1), a type I transmembrane protein, is overexpressed and aberrantly glycosylated in many carcinomas particularly in MM resulting in an antigenically distinct molecule and may be a potential target for specific immunotherapy. In this study, we first designed a unique DNA vaccine, termed MUC1-2-VNTR (various number tandem repeats) to investigate whether the vaccine could specifically suppress tumor growth in a murine multiple myloma model. Our results showed that the constructed DNA vaccine pcDNA3.1-VNTR elicited both humoral and cellular tumor-specific immune responses in the MM mouse model leading to delay in tumor growth and prolonged survival of the mice. Consequently, our study indicates that this DNA vaccine shows promise to be used as a novel strategy for the treatment of MM.
\end{abstract}

\section{Introduction}

Multiple myeloma (MM) is characterized by clonal proliferation of malignant plasma cells. The median survival of MM patients is approximately 3 years (1). Despite recent improvements in the treatment of MM, present therapies still cannot cure the majority of patients with MM and they eventually relapse, mainly due to MRD (minimal residual disease). Many efforts have been made to find new treatments for this disease.

Correspondence to: Dr You Zhang, Department of Hematology, The Second Hospital of Kunming Medical University, Kunming, 650101, P.R. China

E-mail: youzhangmd@yahoo.com.cn

Key words: multiple myeloma, mucin 1, variable number of tandem repeats, DNA vaccine
Tumor vaccine has been one of the developments recently. Mucin 1 (MUC1), a kind of tumor-associated antigen (TAA) and a member of mucin family, is a highly glycosylated type I transmembrane protein normally presented on the luminal surface of secretory glands (2) as well as normal haematopoietic cell lines (3). The extracellular domain of MUC1 primarily consists of 25-125 variable number of tandem repeats (VNTR) of 20 amino acids: VTSAPDTRPAPGSTAPPAHG (4). However, some tumors such as breast, prostate, lung, colon, ovary, pancreas, MM, AML (acute myeloid leukemia), expressed an aberrant form of MUC1 (5-16). The polarized expression of MUC1 is lost and the normally glycosylated protein is overexpressed in hypoglycosylated forms (17), resulting in exposure of cryptic epitopes that have the potential to induce humoral and cellular immunity $(18,19)$. It has also been reported that MUC1 specific immune response could be successfully induced both in mice and human (10-13). Therefore, MUC1 should be an attractive and applicable target strategy to treat MM.

Here, we firstly constructed recombinant vectors encoding MUC1-2-VNTR to investigate the effect of this vaccination on the immune system and its antitumor effects in MM mouse model.

\section{Materials and methods}

Construction of eukaryotic expression vector of MUC1. MUC1-2-VNTR coding gene was used as a research gene, a KOZAK sequence was engineered before the gene HindIII and $X b a \mathrm{I}$ restriction sites. The full length of the gene was $140 \mathrm{bp}$, synthesized by Takara, which subcloned into plamid PUC18, and transformed into E. coli JM109 competent cells. pcDNA3.1/Myc-hisB vector was purchased from Invitrogen, containing multiple cloning site (MCS), BGH reverse priming site, myc epitope, C-terminal polyhistidine tag, SV40 early promoter and origin, polyadenylation signal and ampicillin resistance gene. PUC18/MUC1-2-VNTR plasmid from E. coli JM109 was carried out following the manufacturer's instructions. Then the PUC18/MUC1-2-VNTR plasmid and the pcDNA3.1/Myc-hisB vector were separately digested with $X b a \mathrm{I}$ and HindIII (Takara Biotechnology Co., Ltd., Dalian, 
China), and ligated by T4 DNA ligase (Takara) to construct a plasmid termed pcDNA3.1-2-VNTR/myc-hisB. After that, the ligation mixture was transformed into $E$. coli $\mathrm{DH} 5$ a competent cells, culturing on an LB Petri dish with $100 \mu \mathrm{g} / \mathrm{ml}$ ampicillin at $37^{\circ} \mathrm{C}$ overnight. Ampicillin-resistant transformants were selected to bacterial colony. After the expected bands were observed by electrophoresis gel, the plamid isolated from positive colony was sequenced by Takara.

COS-7 cell transfection. COS-7 cells were routinely cultured in DMEM (Gibco) with $10 \%$ fetal bovine serum (FBS) (HyClone Laboratories, Logan, UT). The day before transfection, $5 \times 10^{5}$ cells $/ \mathrm{ml}$ was plated in each well of a 6 -well plate without antibiotics until 90-95\% confluent at the time of transfection. The mixture of $5 \mu \mathrm{g}$ plasmid GFP, $2.5 \mathrm{~mol} / 1 \mathrm{CaCl}_{2}$, $2 \mathrm{X}$ HBS-HEPES, $5 \mu \mathrm{g}$ plasmid pcDNA3.1-2-VNTR, or empty vector was diluted in $250 \mu \mathrm{l}$ of serum-free medium (SFM) and incubated for $20 \mathrm{~min}$ at room temperature with a mixture of $10 \mu 1$ Lipofectamine 2000 and $250 \mu \mathrm{l}$ SFM and the resultant complex mixture was added to a monolayer of pre-confluent cells seeded in a 6 -well plate. The cells were incubated at $37^{\circ} \mathrm{C}$ and in $5 \% \mathrm{CO}_{2}$ for $6 \mathrm{~h}$, at which time the transfected medium was replaced with fresh and complete one. The cells transfected with vector pcDNA3.1 (+) were generated as a negative control.

COS-7 cell transfection assay by western blot analysis. Forty hours after cell transfection, the supernatant of medium for detection was collected and $1 \times 10^{7}$ cells were prepared in $0.5 \mathrm{ml}$ lysis buffer $(10 \mathrm{mmol} / 1 \mathrm{Tris}-\mathrm{HCl}, 5 \mathrm{mmol} / 1$ EDTA, $50 \mathrm{mmol} / 1 \mathrm{NaCl}, 30 \mathrm{mmol} / 1 \mathrm{Na}_{3} \mathrm{PO}_{4}, 50 \mathrm{mmol} / 1 \mathrm{NaF}$, $0.1 \mathrm{mmol} / 1 \mathrm{Na}_{3} \mathrm{VO}_{4}, 1 \mathrm{mmol} / 1 \mathrm{PMSF}, 5 \mathrm{mg} / \mathrm{ml}$ aprotinin, $0.1 \%$ Triton $\mathrm{X}-100, \mathrm{pH}$ 7.6). The cells were incubated on ice for $15 \mathrm{~min}$ and then centrifuged at $14,000 \mathrm{rpm}$ at $4^{\circ} \mathrm{C}$ for $20 \mathrm{~min}$. The supernatant was then collected followed by denaturing at $100^{\circ} \mathrm{C}$ for $5 \mathrm{~min}$. For western blot analysis, the samples were loaded onto a $12 \%$ SDS-polyacrylamide gel and transfered to a nitrocellulose membrane. The membrane was incubated with a mouse anti-human MUC1 antibody, followed by a horseradish peroxidase-conjugated rat anti-mouse IgG antibody. Then the membrane was reacted with enhanced chemiluminescence detection reagent for $5 \mathrm{~min}$ and exposed to X-ray film at room temperature.

The construction of multiple myeloma cell line 653-MUC1. P3X63Ag.653 cells (mouse myeloma cell line) from CellBank of Chinese Academy of Sciences were grown in RPMI-1640 medium (HyClone) containing 10\% fetal calf serm (FBS, Hyclone), $2 \mathrm{mM}$ L-glutamine, $100 \mathrm{U} / \mathrm{ml}$ penicillin and $100 \mu \mathrm{g} / \mathrm{m}$ streptomycin. The cells were incubated at $37^{\circ} \mathrm{C}$ in a humidified atmosphere of $5 \% \mathrm{CO}_{2}$ and sub-cultured when confluence was reached. The medium was changed every 2-3 days. The MUC1 cDNA (constructed plasmids pcDNA3.1-2VNTR) was transfected into the mouse myeloma cell line using Lipofectamine 2000 Reagent (Invitrogen, Mannheim, Germany) according to the manufacturer's specifications. After selection with G418, clones that expressed MUC1 were obtained by the limiting dilution method. These cell lines were maintained in RPMI-1640 medium containing $10 \%$ fetal bovine serum (FBS).
RNA isolation and RT-PCR. Total RNA was prepared using TRIzol reagent (Invitrogen) as described by the manufacturer. Total RNA was reverse transcribed and amplified using the AccessQuick ${ }^{\mathrm{TM}}$ RT-PCR System (Promega) following the manufacturer's instructions. A pair of PCR primers, 5'-GGGG AGGATTGGGAAGAC-3' (forward) and 5'-CCGAGATAGG GTTGAGTGTT-3' (reverse), was designed according to the sequence of pcDNA3.1-2-VNTR, which amplified a $442 \mathrm{bp}$. $\beta$-actin and 653 cells transfected without pcDNA3.1-2-VNTR was used as controls. The primers, $\beta$-actin, 5 -ATGCCATCCT GCGTCTGGACCTGGC-3' (forward), 5'-AGCATTTGCGGT GCACGATGGAGGG-3' (reverse), amplified a 607 bp. The amplification protocol consisted of a 5-min denaturation at $95^{\circ} \mathrm{C}$ followed by 30 cycles of denaturation at $95^{\circ} \mathrm{C}$ for $50 \mathrm{sec}$, annealing at $54^{\circ} \mathrm{C}$ for $50 \mathrm{sec}$ and $72^{\circ} \mathrm{C}$ for $50 \mathrm{sec}$ and a final extension at $72^{\circ} \mathrm{C}$ for $5 \mathrm{~min}$.

Mice and immunization. The BALB/c mice, female, 7-8 weeks of age, from the Shanghai Laboratory Animals Center, Science Academia of China, were bred in the Animal Lab center (Kunming Medical University, Yunnan). The facility was approved by the Association for Assessment and Accreditation of Laboratory Animal Care International, and all procedures were carried out in accordance with the Guidelines and Regulations for Use and Care of Animals. The mice were divided randomly into 2 groups $(n=10)$. Plasmid DNA $(100 \mu \mathrm{l} / 100 \mu \mathrm{g})$ was injected into the spleen of the mice on Day 0. A booster immunization was given on the fifth week by injecting into each hind quadriceps muscle. The mice treated with the empty plasmid pcDNA3.1 were used as the control. Mice were bled by retroorbital venous orbita at 1, 2, 4, 6 and 8 weeks after the spleen immunization. Sera were collected after centrifugation at $13,000 \mathrm{rpm}$ at $4^{\circ} \mathrm{C}$ for $20 \mathrm{~min}$, then cryopreserved at $-80^{\circ} \mathrm{C}$.

MUC1 antibodies detected by ELISA. Polyvinyl chloride microtiter plates (Costar, Inc., Corning, NY) coated with $500 \mathrm{ng} /$ well MUC1-VNTR was incubated at $4^{\circ} \mathrm{C}$, overnight. The plates were blocked $(0.5 \%$ porcine gelatin, $4 \% \mathrm{BSA}$ in PBS), followed by incubation with diluted mouse sera in serum samples (1:200). Anti-MUC1 antibodies were detected with horseradish peroxidase (HRP)-labeled anti-mouse IgG (H+L) (Pierce Biotechnology, Inc., Rockford, IL) and developed with 3-ethylbenzthiazoline-6-sulfonic acid (ABTS) solution. Absorbance was read at $405 \mathrm{~nm}$. Values (1/titer) were scored as positive for the presence of MUC1 antibodies if the OD readings were at least three-times over PBS control wells.

Proliferative assay by $C C K-8$ method. Four weeks after the second immunization, mice were sacrificed, and single-cell suspension of splenocytes was prepared. They were plated at 500,000/well in 96-well U-bottom plates (\#3799: Costar Co., Cambridge, MA) and incubated with MUC1-VNTR $(10 \mu \mathrm{g} / \mathrm{ml})$ at $37^{\circ} \mathrm{C}$ in $5 \% \mathrm{CO}_{2}$ for 3 days. Sixteen hours before termination of the culturing, $20 \mu \mathrm{l}$ of CellTiter $96^{\circledR}$ AQueous One Solution Reagent (Promega) was added into each well. Stimulation index (SI) was calculated to detect spleen lymphocyte proliferation activity. SI, cpm of the experimental pair/ cpm of negative control. All experiments were performed in 


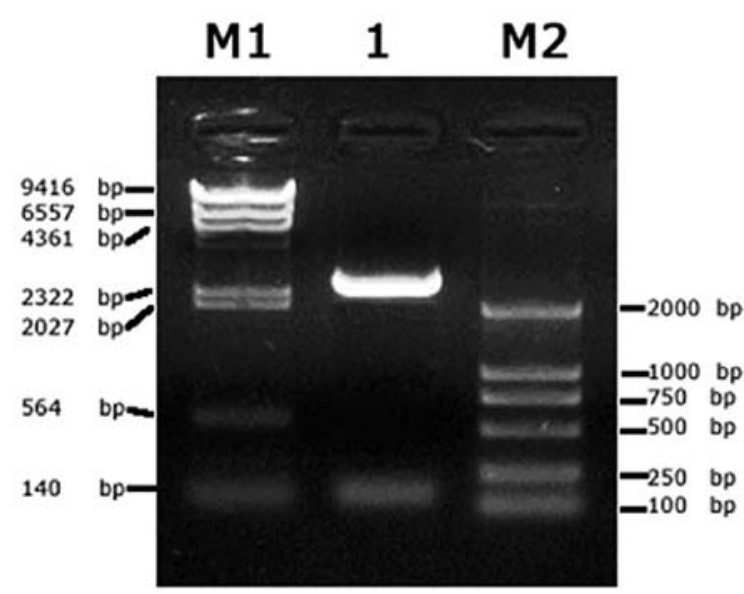

Figure 1. Double restriction of pUC18/MUC1-2-VNTR with $X b a \mathrm{I}$ and HindII. M1, $\gamma$-HindIII DNA marker. 1, pUC18/MUC1-2-VNTR restricted with HindW and XbaI. M2, DNA marker DL2000.

triple samples. ConA and RPMI-1640 were used as positive and negative control, respectively.

Cytotoxicity assay by LDH releasing method. Cytotoxicity of CTL against the target cells was detected by a non-radioactive lactate dehydrogenase (LDH)-releasing assay (CytoTox 96, Promega, USA). Single-cell suspension of splenocytes were collected as effector cells, 653 cells stably transfected with MUC1-VNTR as target cells. The effector cells were co-cultured with target cells at different effector cell/target cell ratios (10:1, 40:1 and 80:1). Then, $1 \times 10^{5}$ target cells per well were mixed with a varying number of effector cells in 96-well round-bottom plates with total volume reaching $200 \mu \mathrm{l}$ and incubated for $4 \mathrm{~h}$ at $37^{\circ} \mathrm{C}$. At the end of the assay, supernatants (50 $\mu \mathrm{l} /$ well) were harvested and transferred to a fresh plate to test $\mathrm{LDH}$ releasing rate according to the manufacturer. The killing activity of CTL cells against tumor cells was calculated with the following formula: killing activity (\%) = (experimental - effector spontaneous - target spontaneous $) /$ (target maximum - target spontaneous) x 100\%. Spontaneous and maximal releases were determined in the presence of either medium or $1 \%$ Triton $\mathrm{X}-100$, respectively.

Animal studies. The BALB/c mice, female, 7-8 weeks old, were purchased from the Shanghai Laboratory Animals Center, Science Academia of China. The mice were randomly divided into 2 groups ( $\mathrm{n}=10$, respectively). The mice were injected with plasmid DNA $(100 \mu \mathrm{l} / 100 \mu \mathrm{g})$ as mentioned above. The empty plasmid pcDNA3.1 was used as control. Seven days after the second immunization, each mouse was inoculated with $1 \times 10^{6}$ 653-MUC1 cells at the interterm of the left anterior leg armpit. The tumor development in individual mouse was monitored every 2-3 days and the tumor volume (in $\mathrm{mm}^{3}$ ) was calculated by the following formula: $0.5 \mathrm{x}$ length $(\mathrm{mm}) \mathrm{x}$ width $(\mathrm{mm})^{2}$. The survival time after the tumor challenge was recorded for 60 days until the death of the mouse.

Statistical analysis. Data are presented as mean \pm SEM. Statistical differences between two groups were evaluated by the unpaired Student's t-test. The survival time was calcu-

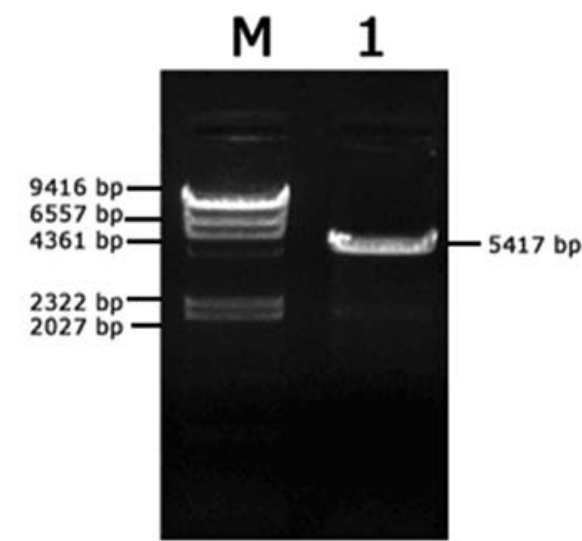

Figure 2. Double restriction of pcDNA3.1/myc-his with HindIII and XbaI. M, $\gamma$-HindIII DNA marker. 1, cDNA3.1/myc-his B restricted with HindIII and $X b a \mathrm{I}$

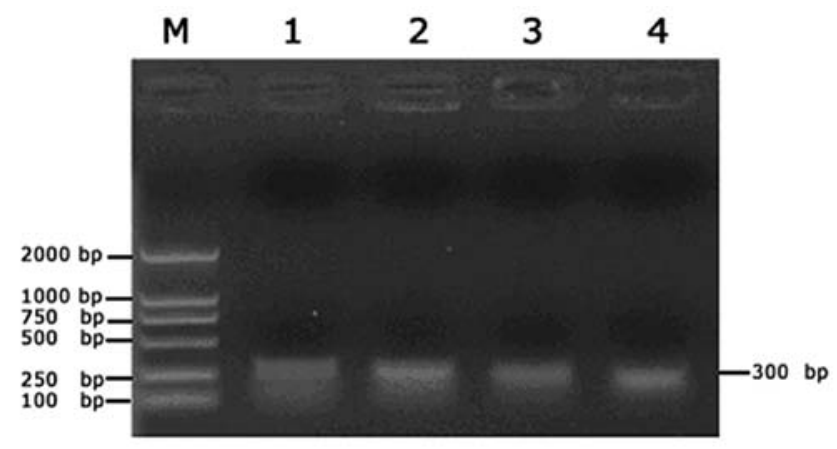

Figure 3. Identification of positive transformed colony by PCR. M, DNA marker DL2000; 1, 2, 3 and 4, the products of PCR.

lated by the Kaplan-Meier method. The survival rates were compared by the log-rank test (SPSS 13.0). P-values $<0.05$ were considered significant.

\section{Results}

Identification of the MUC1-2-VNTR and eukaryotic expressing vector pcDNA3.1/myc-hisB. The MUC1-2-VNTR and eukaryotic expressing vector pcDNA3.1/myc-hisB were cut by the double restriction enzyme XbaI and HindII. Then the enzyme products were electrophoresis using agar gels. The objective fragment 140 bp (Fig. 1) and fragment 5417 bp were obtained (Fig. 2).

Identification of the recombinant plasmids. Four transformants from LB Petri dish with $100 \mu \mathrm{g} / \mathrm{ml}$ ampicillin using T7 promoter/BGHrev primer for PCR were selected, then the PCR products were electrophoresis using the agar gels. The objective fragment $300 \mathrm{bp}$ (Fig. 3) was obtained. The positive transformed colony was selected for DNA sequencing (Fig. 4).

The sequencing includes whole reading frame of pcDNA3.1/myc-his B and the 2-VNTR inserted DNA sequence (underlined). Together, the construction of recombinant plasmid pcDNA3.1-2-VNTR was successful.5'-CCTACTTGG CAGTACATCTACGTATTAGTCATCGCTATTACCATGG GATGCGGTTTTGGCAGTACATCAATGGGCGTGGAGC 


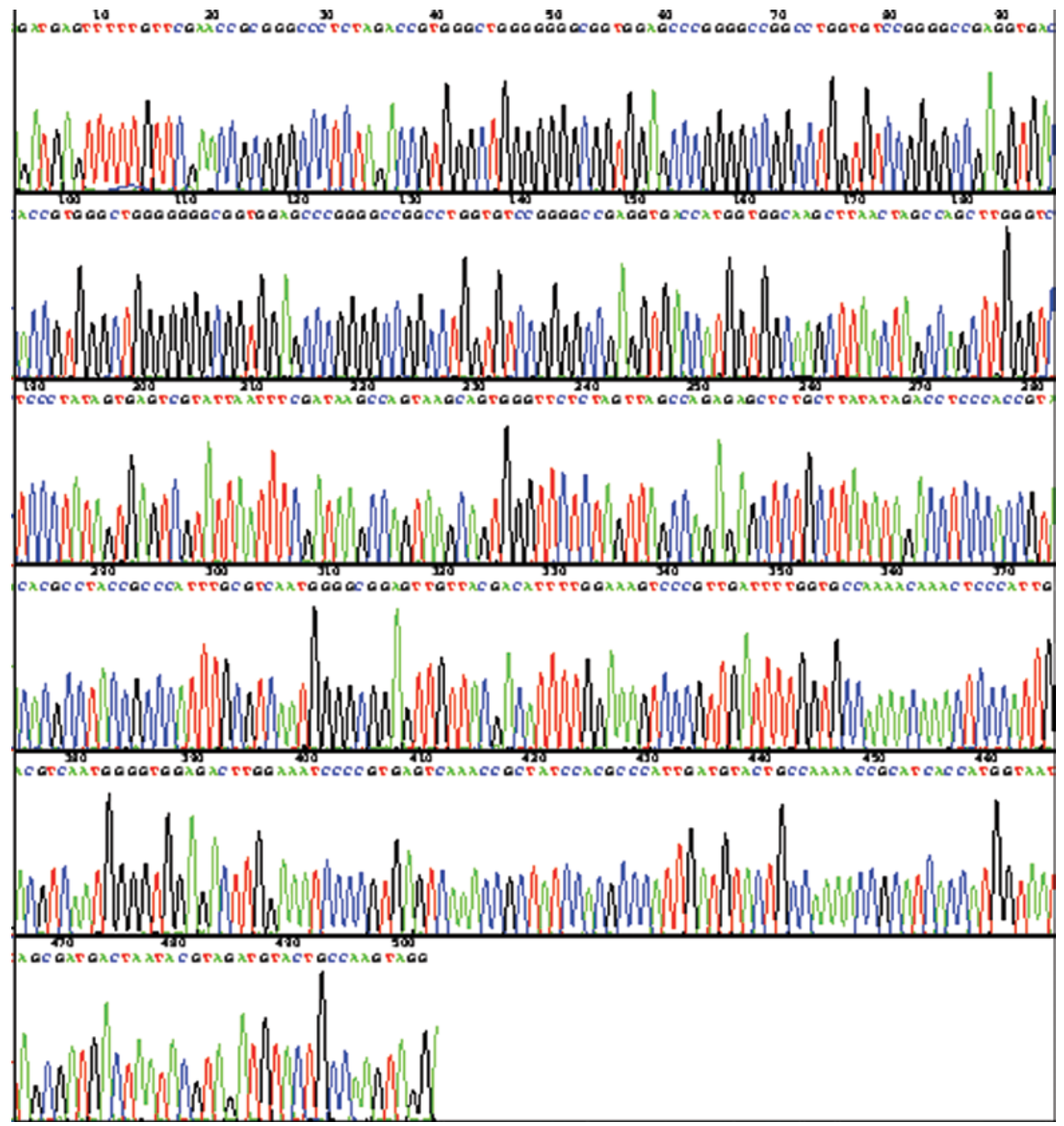

Figure 4. DNA sequencing of recombinant plasmid pcDNA3.1-2-VNTR /myc-his B.

A

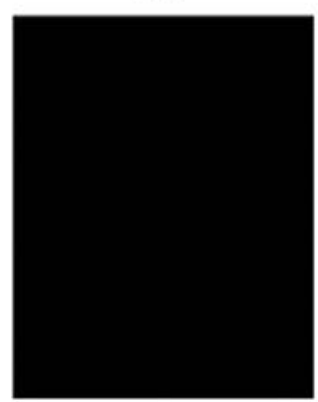

B

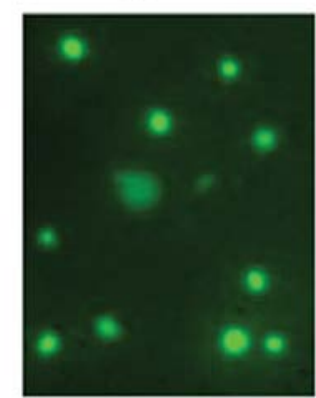

C

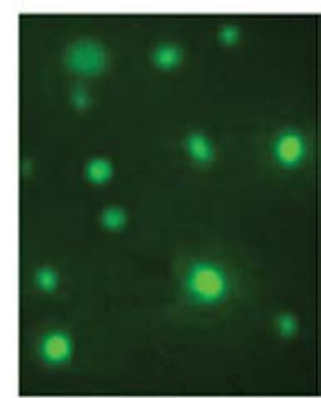

Figure 5. Co-transferred GFP observed by fluorescence microscopy. (A) Untransfected COS-7 cells. (B) COS-7 cells transfected with vacant plasmid pcDNA3.1/myc-his B+GFP. (C) COS-7 cells transfected with recombinant plasmid pcDNA3.1-2VNTR/myc-his B+GFP.

GGTTTGACTCACGGGGATTTCCAAGTCTCCCCCATTG ACGTCAATGGGAGTTTGTTTTGGCCAAAATCAACGG GACTTTCCAAAATGTCGCAACTCCGCCCCATTGACG CAAATGGGCGGAGGCGTGTACGGTGGGAGGTCTATA TAAGCAGAGCTCTCTGGCTAACTAGAGAACCCACTG CTTACTGGCTTATCGAAATTAATACGACTCACTATAG
GGAGACCCAAGCTGGCTAGTTAAGCTTGCCACCAT GGTCACCTCGGCCCCGGACACCAGGCCGGCCCCGG GCTCCACCGCCCCCCCAGCCCACGGTGTCACCTCG GCCCCGGACACCAGGCCGGCCCCGGGCTCCACCGC CCCCCCAGCCCACGGTCTAGAGGGCCCGCGGTTCG AACAAAAACTCATC-3'. 


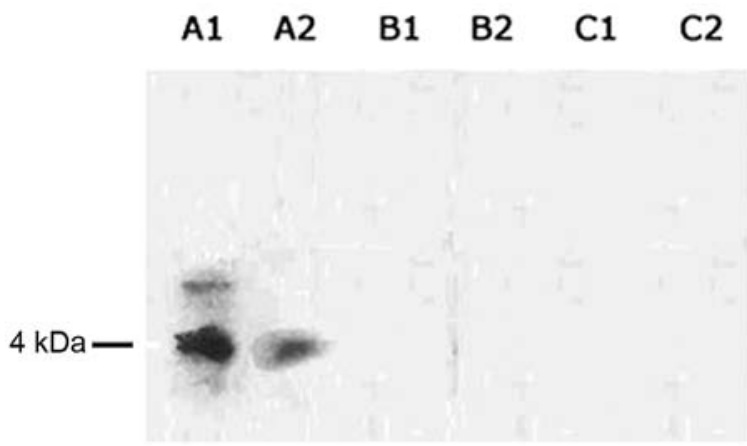

Figure 6. Results detected by western blot analysis. A1, COS-7 cell lysate transfected with recombinant plasmid pcDNA3.1-2-VNTR/myc-hisB. A2, cultured COS-7 cell culture supernatant transfected with recombinant plasmid pcDNA3.1-2-VNTR/myc-his B. B1, COS-7 cell lysate transfected with vacant plasmid pcDNA3.1/myc-his B. B2, COS-7 cell culture supernatant transfected with vacant plasmid pcDNA3.1/myc-his B. C1, untransfected COS-7 cell lysate. C2, untransfected COS-7 cell culture supernatant.

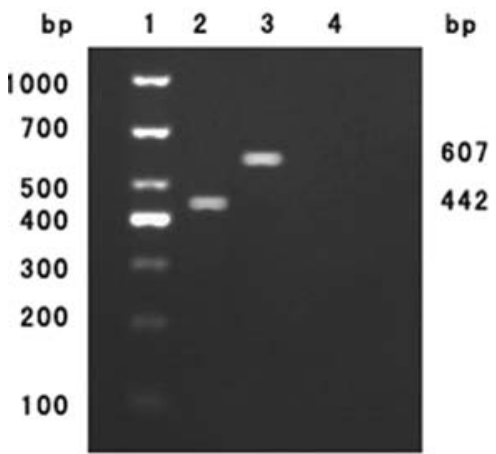

Figure 7. Detection of transfected cells by RT-PCR. Lane 1, DL1, 000 DNA marker; lane 2, 653 cells transfected with pcDNA3.1-2-VNTR; lane 3, $\beta$-actin; lane 4, 653 cells transfected without pcDNA3.1-2-VNTR.

Cotransfected COS-7 cells with pcDNA3.1+GFP. COS-7 cells were co-cultured with vacant plasmid pcDNA3.1/mychisB+GFP or recombinant plasmid pcDNA3.1-2-VNTR/ myc-hisB+GFP for 2 days, followed by the detection of fluorescence microscopy. The results showed that recombinant pcDNA3.1/myc-his B and pcDNA3.1-2V-NTR-hisB were successfully transfected into COS-7 cells (Fig. 5).

Detection of expression of pcDNA-2-VNTR protein by western blot analysis. The result of western blot analysis showed MUC1 protein was expressed in the cells transfected with pcDNA plamid. After probing with antibodies against VNTR, the molecular weight of objective band was $4 \mathrm{kDa}$ (Fig. 6).

Monoclonal cell line verification by RT-PCR. P3X63Ag.653 transfected by pcDNA3.1-2-VNTR showed much stronger expression of VNTR. Band 2 was about 121 bp as expected, whereas, we detected no expression of VNTR in the control group (P3X63Ag.653 transfected by vector pcDNA3.1(+) (Fig. 7).

Elevated level of specific antibodies in the sera of vaccinated mice. Mice were bled by retro-orbital venous in week 1, 2, 4, 6 and 8 after the spleen immunization. Specific anti-MUC1

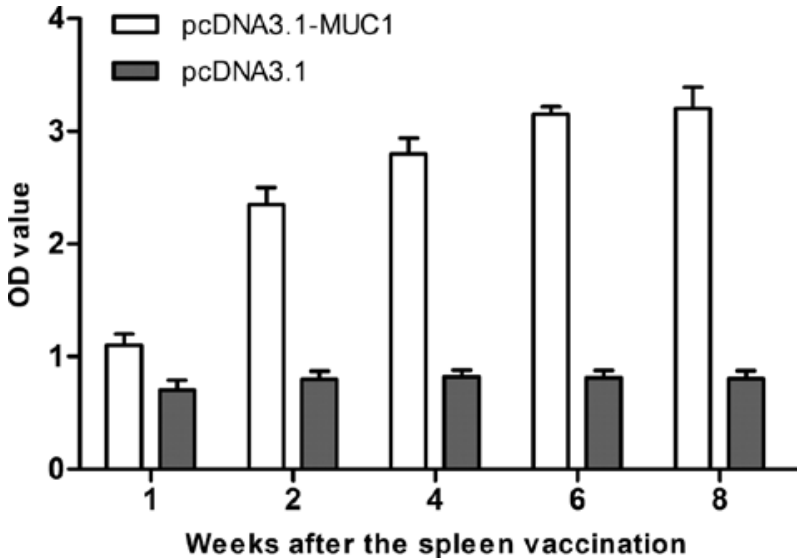

Figure 8. Induction of specific antibody responses.

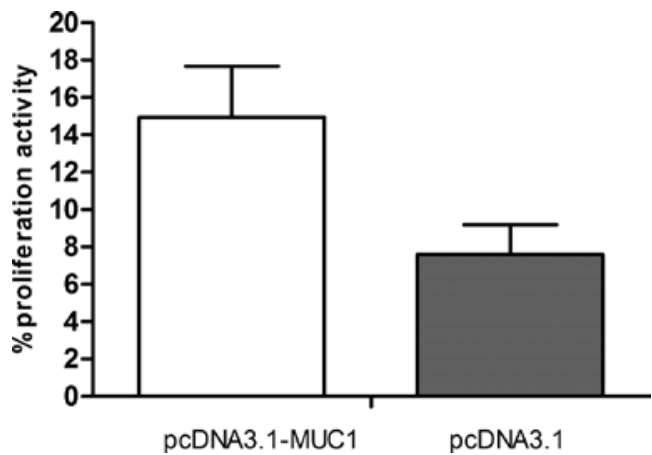

Figure 9. Effects of pcDNA3.1-MUC1 on the proliferation of lymphocytes.

antibody can be detected in the first week after the spleen vaccination, and the antibody level reached a peak in the eighth week. Compared with the empty vector group, significantly higher titres of anti-MUC1 antibodies were detected in the sera of pcDNA3.1-VNTR-vaccinated mice $(\mathrm{P}<0.01)$ (Fig. 8). The result indicated that pcDNA3.1-VNTR can efficiently induce a specific humoral immune response via induction of specific antibodies.

Induction of specific antibody response. Mice were immunized with pcDNA3.1-VNTR or pcDNA3.1 and sera were harvested in week 1, 2, 4, 6 and 8 after the spleen immunization. The MUC1-specific IgG antibodies in sera of different groups were determined at a 1:200 dilution by ELISA. As shown in Fig. 8, relatively higher titres of anti-MUC1 antibodies were detected in the serum of pcDNA3.1-VNTR vaccinated mice compared to that in the sera of the empty vector vaccinated groups in week 1, 2, 4, 6 and $8(\mathrm{P}<0.01)$.

Effects of pcDNA3.1-MUC1 on the proliferation of lymphocytes. To test the effect of pcDNA3.1-MUC1 vaccine on the proliferation of lymphocytes, lymphocytes were isolated from the spleens of immunized mice four weeks after the second immunization. We chose to optimize and qualify the colorimetric cell proliferation assay using MTS (aqueous soluble form of the tetrazolium salt based assay) with the Cell Titer96 ${ }^{\circledR}$ Aqueous One assay kit from Promega Corp. The results showed that proliferation activity of lymphocytes from mice 


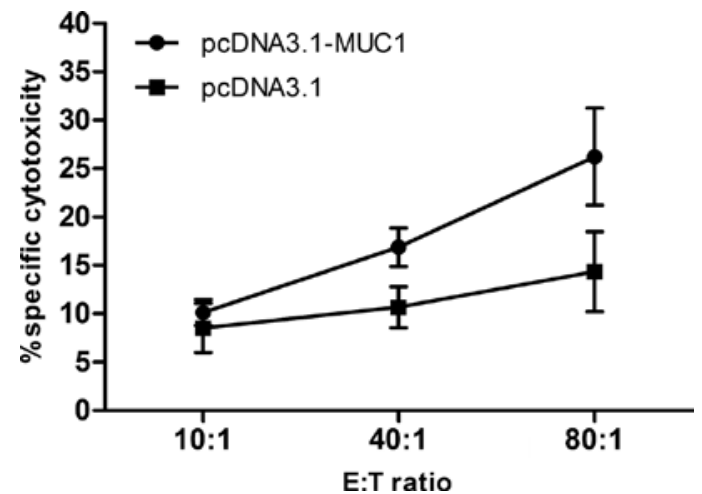

Figure 10. Induction of specific CTL cytotoxity by pcDNA3.1-MUC1 vaccine. The following are the treatment groups: group A, pcDNA3.1-VNTR (solid diamond); group B, pcDNA3.1 (solid square). Splenocytes from immunized mice were isolated four weeks after the second immunization CTLs were from mice immunized with pcDNA3.1-VNTR or pcDNA3.1. 653-MUC1 cells were used as targets. The CTLs induced by the pcDNA3.1VNTR could specifically kill the 653-MUC1 cells.

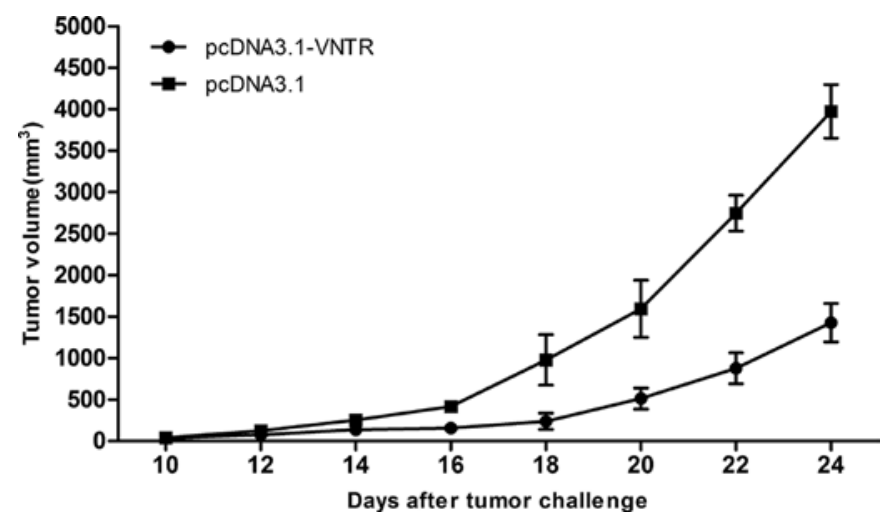

Figure 11. Effect of tumor vaccine on the tumor growth. The following are the treatment groups: group A, pcDNA3.1-VNTR $(n=10)$ (solid diamond); group B, pcDNA3.1 $(n=10)$ (solid square). Mice were immunized with pcDNA3.1VNTR or the empty vector pcDNA3.1 as indicated in the legends, followed by challenge with $653-\mathrm{MUC1}$ tumor cells. Each plasmid was administered at $100 \mu \mathrm{g}$. The mean tumor volume of the mice immunized with pcDNA3.1VNTR was significantly smaller than that in the mice in the control group (mean \pm SEM. $\mathrm{P}<0.0001)$.

vaccinated with recombinant plasmid group was significantly higher than that of the empty vector treated mice $(14.94 \pm 2.74$ vs. 7.61 $\pm 1.59, \mathrm{P}<0.01$ ) (Fig. 9).

Splenocytes from immunized mice were isolated four weeks after the second immunization. As shown in Fig. 9, proliferation activity of lymphocytes from pcDNA3.1-VNTR immunized mice was significantly higher than that of the pcDNA3.1 immunized mice.

Effect of pcDNA3.1-VNTR on cytotoxicity responses in vitro. To determine whether immunization with pcDNA3.1-VNTR could induce strong CTL response, we detected the cytotoxity response by non-radioactive LDH-releasing assay. Effector cells were co-cultured with target cells in different ratios 80:1, 40:1 and 10:1. There was significant difference in the ratios of both $80: 1$ and $40: 1$, with the killing rate of $26.23 \pm 5.02$ and $16.88 \pm 1.98$, respectively, compared with $14.36 \pm 4.13$ and $10.67 \pm 2.11$ in the empty vector control group. However,

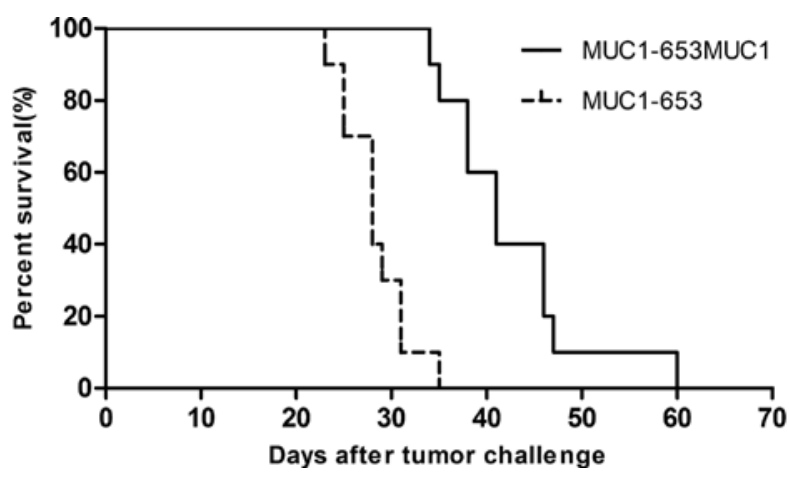

Figure 12. Survival curves from two groups after tumor challenge. The following are the treatment groups: group A, MUC1-653 $(n=10)$ (dash line); group B, MUC1-653MUC1 ( $\mathrm{n}=10$ ) (bold line). Mice were immunized with pcDNA3.1-VNTR, the empty vector pcDNA3.1 as indicated in the legends, followed by challenge with MUC1-653 tumor cells. Mice were monitored for tumor development until death. As shown in the figure, the mice immunized with pcDNA3.1-VNTR survived longer compared to the mice in the control groups $(\mathrm{P}<0.01)$.

there was no significant difference in the ratio of 10:1 (E:T) $(10.30 \pm 1.65$ vs. $8.98 \pm 2.16, \mathrm{P}>0.05)$ between the two groups (Fig. 10). These results confirmed that pcDNA3.1-VNTR are capable of inducing MUC1-specific CTL effect in vitro.

Effect of pcDNA3.1-VNTR vaccine on multiple myeloma in vivo. To determine whether pcDNA3.1-VNTR vaccine is effective in suppressing multiple myeloma in vivo, cohorts of mice were inoculated with pcDNA3.1-VNTR twice before challenging with $1 \times 10^{6}$ pcDNA3.1-VNTR or pcDNA3.1, respectively. As shown in Fig. 11, tumor of the mice immunized with pcDNA3.1-VNTR grew slower compared with mice treated with pcDNA3.1. Furthermore, immunized mice were tumor-challenged and monitored for tumor growth for 60 days. As shown in the Fig. 12, the mice immunized with pcDNA3.1-VNTR survived longer than mice in control group ( $P<0.01$ by log-rank test). Mean survival time of pcDNA3.1VNTR immunized mice was 41 days, higher than the 28 days of pcDNA3.1 immunized mice, demonstrating that the pcDNA3.1-VNTR vaccine could efficiently induce suppressive effect on tumor growth.

\section{Discussion}

MM is still considered as an incurable malignancy. Many efforts have been made to find a new way to treat the disease. In general, the growth of malignant cells in MM is relatively lower than that of other hematologic malignant cells such as leukemic cells, which may result in the low sensitivity to chemotherapy but, at the same time, may lead to be more suitable for the use of immunotherapy especially after CR (complete remission) to eliminate the MRD (minimal residual disease).

MUC1, a tumor-associated antigen (TAA), is composed of a large ectodomain that is heavily glycosylated through GalNAc O-linkages, a single-pass transmembrane region, and an intracellular cytoplasmic tail (CT) (20-22). The extracellular domain of MUC1 primarily consists of 25-125 various number tandem repeats (VNTR) of 20 amino acids and varies among individuals or directly derived tissues (4), which is 
the most immunogenic part of MUC1 and many T and B cell antigen recognition sites generated to date almost exclusively recognize epitopes in that immunogenic region (23-25). Thus, MUC1-VNTR sequences may be expected to induce host immune specific CTL responses and as a possible target treatment approach for MM.

In the mid-80s MUC1 was considered to be a tumor marker when it was detected in the serum of most breast cancer patients (26). The presence of MUC1 was demonstrated in subgroups of human lymphomas, including plasma cell myeloma in the early 1980s $(27,28)$.

Non-MHC-restricted cytotoxic T cell activity has been described on multiple myeloma cells by Takahashi et al (15). In a published study, $44 \%$ of myeloma patients exhibited elevated frequencies of MUC1-specific CD8 T cells in peripheral blood as well as bone marrow (29).

Recently, MUC1 immunization studies mainly focus on peptides vaccine (30-34), virus vaccine (35-37), dendritic cells (DC) and DC/tumor cell fusions vaccine (38-42), RNA vaccine (38) and DNA vaccines (43-45). MUC1-DNA immunization has been actively investigated since the demonstration of tumor protective immune response after intramuscular injection of naked MUC1-DNA (46).

In our previous studies, we have confirmed that MUC1 was highly expressed in MM patients by immunochemistry, ELISA and RT-PCR (47-49). On the other hand, the low tumor burden in the $\mathrm{CR}$ patients with $\mathrm{MM}$ and the low growth rate of MM cells may benefit the most from the DNA vaccine for treatment of MM.

Based on the evidence, we supposed that the approach of using MUC1 as a target to further eradiate MRD of MM may be reasonable. The eukaryotic expression vector pcDNA3.1/ myc-hisB used in this study is typical and efficient vector which can be expressed in the majority of mammals. DNA vaccines have been widely investigated in a variety of disease models and clinical trials including autoimmune disease, infectious disease as well as carcinoma. However, we realized that DNA vaccines may generate weak immune response without any booster or modification. So, in order to enhance the immunogenicity of MUC1, we designed a unique 2-fold VNTR sequence (2-VNTR) as a target antigen to construct a recombinant plasmid and an optimized KOZAK sequence, at the same time, engineered to the start codon region of the VNTR, which has been proven to significantly promote the gene expression in eukaryotic cells (50). This MUC1-DNA vaccine was confirmed to be successfully constructed by both cell colony PCR and DNA sequencing. Furthermore, both transient transfection and western blot analysis demonstrated that pcDNA3.1-VNTR induced expression of MUC1 protein.

To investigate the humoral and cellular immune responses induced by MUC1-VNTR vaccine in multiple myeloma, we used pcDNA3.1-VNTR immunized female BALB/C mice to detect both of them. Detection of specific antibodies by ELISA confirmed that humoral immune responses were elicited by pcDNA3.1-VNTR and cytotoxicity assay showed that splenocytes from the pcDNA3.1-VNTR immunized mice could specifically kill the 653-MUC1 tumor cells.

In the suppression experiment of the model, we found that the median survival of vaccinated mice was significantly longer than that of the control group. In addition, the tumor growth of the mice was markedly suppressed. These results showed that MUC1-2-VNTR vaccine may effectively inhibit or kill MM tumor cells in vivo and potentially to eliminate the MRD of MM, at the end, to cure the disease. The mechanism involved in this strong effect may be mainly due to the unique design of the 2-fold VNTR sequence which greatly enhance the specific CTL effect and lymphocyte proliferation as well as boosting specific anti-MUC-1 antibody produced.

However, the vaccine may not be strong enough to abrogate all the MM tumor cells, as all the mice that received pcDNA3.1-2-VNTR vaccine still died within 60 days. Future work might be focused on further enhancing the effectiveness of the vaccine by, for example, designing three-fold VNTR sequences, or to transfect siRNA (small interfering RNA) into DC (dentritic cell) pulsed with MUC1-2-VNTR to silence the expression of SOCS1 (suppressor of cytokine signaling).

Our study, for the first time, uses pcDNA3.1-2-VNTR vaccine to suppress the growth of MM cells in mouse model demonstrating that our hypothesis above may be correct. It may provide a potential therapeutic approach to treat MM patients in the future.

\section{Acknowledgements}

This study is supported by grants from the National Natural Science Foundation of China (no. 30760080).

\section{References}

1. Jemal A, Siegel R, Ward E, Hao Y, Xu J and Thun MJ: Cancer statistics. CA Cancer J Clin 59: 225-249, 2009.

2. Patton S, Gendler SJ and Spicer AP: The epithelial mucin, MUC1, of milk, mammary gland and other tissues. Biochim Biophys Acta 1241: 407-423, 1995.

3. Berois N, Varangot M, Aizen B, et al: Molecular detection of cancer cells in bone marrow and peripheral blood of patients with operable breast cancer. Comparison of CK19, MUC1 and CEA using RT-PCR. Eur J Cancer 36: 717-723, 2000.

4. Seregni E, Botti C, Massaron S, et al: Structure, function and gene expression of epithelial mucins. Tumori 83: 625-632, 1997.

5. Gendler S, Taylor-Papadimitriou J, Duhig T, Rothbard J and Burchell J: A highly immunogenic region of a human polymorphic epithelial mucin expressed by carcinomas is made up of tandem repeats. J Biol Chem 263: 12820-12823, 1988.

6. Siddiqui J, Abe M, Hayes D, Shani E, Yunis E and Kufe D: Isolation and sequencing of a cDNA coding for the human DF3 breast carcinoma-associated antigen. Proc Natl Acad Sci USA 85: $2320-2323,1988$

7. Girling A, Bartkova J, Burchell J, Gendler S, Gillett C and Taylor-Papadimitriou J: A core protein epitope of the polymorphic epithelial mucin detected by the monoclonal antibody SM-3 is selectively exposed in a range of primary carcinomas. Int $\mathbf{J}$ Cancer 43: 1072-1076, 1989.

8. Brossart P, Heinrich KS, Stuhler G, et al: Identification of HLA-A2-restricted T-cell epitopes derived from the MUC1 tumor antigen for broadly applicable vaccine therapies. Blood 93: 4309-4317, 1999

9. Duperray C, Klein B, Durie BG, et al: Phenotypic analysis of human myeloma cell lines. Blood 73: 566-572, 1989.

10. Mark AS and Mangkornkanok M: B-cell lymphoma marking only with anti-epithelial membrane antigen. Cancer 63: 2152-2155, 1989.

11. Delsol G, Al Saati T, Gatter KC, et al: Coexpression of epithelial membrane antigen (EMA), Ki-1, and interleukin-2 receptor by anaplastic large cell lymphomas. Diagnostic value in so-called malignant histiocytosis. Am J Pathol 130: 59-70, 1988.

12. Apostolopoulos V and McKenzie IF: Cellular mucins: targets for immunotherapy. Crit Rev Immunol 14: 293-309, 1994. 
13. Finn OJ, Jerome KR, Henderson RA, et al: MUC-1 epithelial tumor mucin-based immunity and cancer vaccines. Immunol Rev 145: 61-89, 1995.

14. Barnd DL, Lan MS, Metzgar RS and Finn OJ: Specific, major histocompatibility complex-unrestricted recognition of tumorassociated mucins by human cytotoxic T cells. Proc Natl Acad Sci USA 86: 7159-7163, 1989.

15. Takahashi T, Makiguchi Y, Hinoda Y, et al: Expression of MUC1 on myeloma cells and induction of HLA-unrestricted CTL against MUC1 from a multiple myeloma patient. J Immunol 153 2102-2109, 1994.

16. Noto H, Takahashi T, Makiguchi Y, Hayashi T, Hinoda $\mathrm{Y}$ and Imai $\mathrm{K}$ : Cytotoxic $\mathrm{T}$ lymphocytes derived from bone marrow mononuclear cells of multiple myeloma patients recognize an underglycosylated form of MUC1 mucin. Int Immunol 9: 791-798, 1997.

17. Taylor-Papadimitriou $\mathbf{J}$ and Epenetos AA: Exploiting altered glycosylation patterns in cancer: progress and challenges in diagnosis and therapy. Trends Biotechnol 12: 227-233, 1994.

18. Nuti M, Rughetti A, Turchi V, Apollonj-Ghetti C, Scambia G and Frati L: Human B cell immune response to selected epitopes of the polymorphic epithelial mucin (PEM) in cancer patients. In Vivo 7: 645-647, 1993

19. Sorensen AL, Reis CA, Tarp MA, et al: Chemoenzymatically synthesized multimeric Tn/STn MUC1 glycopeptides elicit cancer-specific anti-MUC1 antibody responses and override tolerance. Glycobiology 16: 96-107, 2006.

20. Hareuveni M, Tsarfaty I, Zaretsky J, et al: A transcribed gene, containing a variable number of tandem repeats, codes for a human epithelial tumor antigen. cDNA cloning, expression of the transfected gene and over-expression in breast cancer tissue. Eur J Biochem 189: 475-486, 1990.

21. Lan MS, Batra SK, Qi WN, Metzgar RS and Hollingsworth MA: Cloning and sequencing of a human pancreatic tumor mucin cDNA. J Biol Chem 265: 15294-15299, 1990.

22. Merlo GR, Siddiqui J, Cropp CS, et al: Frequent alteration of the DF3 tumor-associated antigen gene in primary human breast carcinomas. Cancer Res 49: 6966-6971, 1989.

23. Silverman HS, Sutton-Smith M, McDermott K, et al: The contribution of tandem repeat number to the O-glycosylation of mucins. Glycobiology 13: 265-277, 2003.

24. Imai K: New mucin core protein genes and their clinical application. Hokkaido Igaku Zasshi 71: 139-143, 1996 (In Japanese).

25. Quinlin IS, Burnside JS, Dombrowski KE, Phillips CA, Dolby N and Wright SE: Context of MUC1 epitope: immunogenicity. Oncol Rep 17: 453-456, 2007.

26. Hilkens J, Kroezen V, Bonfrer JM, De Jong-Bakker M and Bruning PF: MAM-6 antigen, a new serum marker for breast cancer monitoring. Cancer Res 46: 2582-2587, 1986.

27. Delsol G, Gatter KC, Stein H, et al: Human lymphoid cells express epithelial membrane antigen. Implications for diagnosis of human neoplasms. Lancet 2: 1124-1129, 1984.

28. Sloane JP, Hughes F and Ormerod MG: An assessment of the value of epithelial membrane antigen and other epithelial markers in solving diagnostic problems in tumour histopathology. Histochem J 15: 645-654, 1983.

29. Choi C, Witzens M, Bucur M, et al: Enrichment of functional CD8 memory T cells specific for MUC1 in bone marrow of patients with multiple myeloma. Blood 105: 2132-2134, 2005

30. Ding L, Lalani EN, Reddish M, et al: Immunogenicity of synthetic peptides related to the core peptide sequence encoded by the human MUC1 mucin gene: effect of immunization on the growth of murine mammary adenocarcinoma cells transfected with the human MUC1 gene. Cancer Immunol Immunother 36 : $9-17,1993$.

31. Zhang S, Graeber LA, Helling F, et al: Augmenting the immunogenicity of synthetic MUCl peptide vaccines in mice. Cancer Res 56: 3315-3319, 1996.

32. Gilewski T, Adluri S, Ragupathi G, et al: Vaccination of high-risk breast cancer patients with mucin-1 (MUC1) keyhole limpet hemocyanin conjugate plus QS-21. Clin Cancer Res 6: 1693-1701, 2000
33. Soares MM, Mehta V and Finn OJ: Three different vaccines based on the 140-amino acid MUC1 peptide with seven tandemly repeated tumor-specific epitopes elicit distinct immune effector mechanisms in wild-type versus MUC1-transgenic mice with different potential for tumor rejection. J Immunol 166: 6555-6563, 2001.

34. Westerlind U, Schroder H, Hobel A, et al: Tumor-associated MUC1 tandem-repeat glycopeptide microarrays to evaluate serum- and monoclonal-antibody specificity. Angew Chem Int Ed Engl 48: 8263-8267, 2009.

35. Acres B, Apostolopoulos V, Balloul JM, et al: MUC1-specific immune responses in human MUC1 transgenic mice immunized with various human MUC1 vaccines. Cancer Immunol Immunother 48: 588-594, 2000.

36. Scholl SM, Balloul JM, Le Goc G, et al: Recombinant vaccinia virus encoding human MUC1 and IL2 as immunotherapy in patients with breast cancer. J Immunother 23: 570-580, 2000.

37. Rochlitz C, Figlin R, Squiban P, et al: Phase I immunotherapy with a modified vaccinia virus (MVA) expressing human MUC1 as antigen-specific immunotherapy in patients with MUC1positive advanced cancer. J Gene Med 5: 690-699, 2003.

38. Koido S, Kashiwaba M, Chen D, Gendler S, Kufe D and Gong J: Induction of antitumor immunity by vaccination of dendritic cells transfected with MUC1 RNA. J Immunol 165: 5713-5719, 2000.

39. Gong J, Chen D, Kashiwaba M, et al: Reversal of tolerance to human MUC1 antigen in MUC1 transgenic mice immunized with fusions of dendritic and carcinoma cells. Proc Natl Acad Sci USA 95: 6279-6283, 1998.

40. Tanaka Y, Koido S, Chen D, Gendler SJ, Kufe D and Gong J: Vaccination with allogeneic dendritic cells fused to carcinoma cells induces antitumor immunity in MUC1 transgenic mice. Clin Immunol 101: 192-200, 2001

41. Pecher G, Haring A, Kaiser L and Thiel E: Mucin gene (MUC1) transfected dendritic cells as vaccine: results of a phase I/II clinical trial. Cancer Immunol Immunother 51: 669-673, 2002.

42. Chen D, Xia J, Tanaka Y, et al: Immunotherapy of spontaneous mammary carcinoma with fusions of dendritic cells and mucin 1-positive carcinoma cells. Immunology 109: 300-307, 2003.

43. Graham RA,Burchell JM, Beverley P and Taylor-Papadimitriou J: Intramuscular immunisation with MUC1 cDNA can protect C57 mice challenged with MUC1-expressing syngeneic mouse tumour cells. Int J Cancer 65: 664-670, 1996.

44. Johnen H, Kulbe H and Pecher G: Long-term tumor growth suppression in mice immunized with naked DNA of the human tumor antigen mucin (MUC1). Cancer Immunol Immunother 50: 356-360, 2001.

45. Plunkett T, Graham R, Correa I, et al: Protection against MUC1 expressing mouse tumours by intra-muscular injection of MUC1 cDNA requires functional $\mathrm{CD}^{+}$and $\mathrm{CD}^{+} \mathrm{T}$ cells but does not require the MUC1 tandem repeat domain. Int J Cancer 109: 691-697, 2004

46. Tang DC, DeVit M and Johnston SA: Genetic immunization is a simple method for eliciting an immune response. Nature 356 : 152-154, 1992.

47. Wei H, Zhang Y and Yang L: The expression of MUC1 gene in $\mathrm{mm}$ and its clinical significance. J Leukemia Lymphoma 14 30-32, 2005 (In Chinese)

48. Yang H, Zhang Y, Yang L, Gao C and Wang X: The expression of MUC1 gene in hematological malignances and its clinical significance. J Leukemia Lymphoma 15: 438-441, 2006 (In Chinese).

49. Wang X, Zhang Y, Yang $\mathrm{H}$, Gao C and Mou H: The expression of serum MUC1 in multiple myeloma. J Leukemia Lymphoma 17: 231-233, 2008 (In Chinese)

50. Kozak M: An analysis of 5'-non-coding sequences from 699 vertebrate messenger RNAs. Nucleic Acids Res 15: 8125-8148, 1987. 